\title{
Intelligent Method for Face Recognition of Infant
}

\author{
Shrikant Tiwari \\ Department of Computer \\ Engineering \\ Indian Institute of Technology \\ Banaras Hindu University \\ Varanasi, India-221005
}

\author{
Aruni Singh \\ Department of Computer \\ Engineering \\ Indian Institute of Technology \\ Banaras Hindu University \\ Varanasi, India-221005
}

\author{
Sanjay Kumar Singh \\ Department of Computer \\ Engineering \\ Indian Institute of Technology \\ Banaras Hindu University \\ Varanasi, India-221005
}

\begin{abstract}
Mixing, abduction and illegal adoption of infant is a global challenge and the research done to solve this problem is minimal. Traditional methods of biometric and non biometric techniques have their own limitations and fail to provide required level of security. The task of face recognition has been actively researched in recent year and automatic face recognition of infant is not reported in the literature. The contributions of this research are: (i) Design and implementation of intelligent face recognition technique to recognize infant. (ii) Preparing an infant face database of 150 individuals with slight variations in pose, illumination; expression and quality. The results on the infant database are very encouraging.
\end{abstract}

\section{Keywords}

Face Recognition, Infant, Neural network, Intelligent

\section{INTRODUCTION}

Swapping, mixing and abduction of infant babies are global problem and has become a challenge for law enforcement agencies to curb this menace. According to study in United States, every year approximately 1,00,000 - 5,00,000 infant are switched unknowingly. Apart from these incidents, there are instances of abducting babies and illegal adoption [1]. National Centre for Missing and Exploited Children, 270 cases of infant abduction has been reported in the United States [2]. Another study performed in United States concluded that, out of 34 infants that are admitted to a neonatal intensive care unit at any given day, there is $50 \%$ chance of incorrect identification [3]. In order to reduce the mixing of infant, hospitals have devised several rules. One of the traditional methods to identify the infants is the use of ID bracelets which are put on babies hands/legs right after birth, but this has not been able to prevent swapping of the babies.

Many hospitals tried to use biometric for the identification of infant but could not get satisfactory result as in the case of adults. Biometrics is the science of establishing human identity based on the physical or behavioral characteristics of an individual [4]. Several such traits such as face, iris, hand geometry and fingerprint have been extensively studied in the literature and have been incorporated in both government and civilian identity management applications but none of them are used for infant identification. An ideal biometric trait should exhibit the following properties: universality, uniqueness, permanence, collectability, acceptability, performance, and robustness to spoofing and circumvention [4]. In practice, no single biometric trait can be considered to be ideal. So, the choice of a biometric trait is largely governed by the type of application and the target population interacting with the biometric system. It has been generally understood that the face biometric is preferred when minimal user cooperation (e.g., in surveillance applications) is required, and fingerprint or iris are preferred when high recognition accuracy is required.

Face recognition is important research problem spanning numerous fields and disciplines. Face recognition by humans has its own limitations because in addition to race, face recognition abilities can also vary for faces of different age group. It has been demonstrated that older adults have better recognition memory for faces of older adults compared with faces of younger adults and children $[5,6,7]$. Children have also shown better recognition memory for faces of children compared with faces of younger and older adults [8]. This research involved a multidisciplinary team composed of medical doctors, computer scientists and nurses.

The rest of the paper is organized as follows: existing methods is introduced Section II. Issues involved in face recognition of infants are explained in Section III. Infant database description is in section IV and the proposed methodology and experimental results are reported in Section V and section VI. Finally the Conclusions of this paper are given in Section VII.

\section{EXISTING METHODS}

Different methods have been used by medical doctors to identify infant and some of the important methods are discussed below. Hospitals have devised several methods to recognize infant and two of the most popular methods are foot printing and ID bracelets. About $90 \%$ of the hospitals in United States perform foot printing of the babies within 2 hours of their birth. This is performed on infant recognition form provided by the hospitals on which foot print of the infant and fingerprint of the mother are collected at the time of birth. The footprints are collected by applying ink on the foot with a cylinder and then printed on infants recognition form along with mother fingerprint. The applicability and reliability of using footprints for infant identification has been studied by medical and computer scientist.

The analysis done by Sheppard et al. demonstrates that out of 51 infants the fingerprint experts were able to identify only 10 infants correctly using footprint $[9,10,11,12]$. Similar study was done by Pela et. al. on 1917 footprints collected by trained staff of a hospital in Brazil. They concluded that none of the images provided sufficient information to perform accurate identification $[12,13]$. Further biometrics researchers explored the applicability of other biometrics feature like fingerprint/ palmprint and ear for recognition of infants. The work done by Weingaertner using high recognition sensor for capturing the foot and palmprint of infant, here two images of 106 infants were captured: one within 24 hours of birth and another at around 48 hours. The identification accuracy of $67.7 \%$ and $83 \%$ were obtained using foot prints and palm prints respectively by fingerprint experts [14]. 
Further several reports and studies have stated that capturing the image of finger/palm/footprint of infants is very challenging as it is difficult to hold their hands and legs still. The applicability of ear recognition on a database 206 infants by Fields et al. demonstrated that ear can be used for verification between two children [15]. In all the methods for identifying infants, no research has evaluated the performance of automatic identification/ verification. Other biometric modalities that have been extensively studied for adults are face and iris recognition $[16,17]$. Although iris recognition for adults yields very high accuracy for infants, it is very difficult to capture iris patterns [17].

Very few articles referring to the use of fingerprints or palmprints on infants were found. Worth mentioning is Sir F. Galton's work [11] where he presented a study of infant fingerprinting with ink and paper, concluding that fingerprints taken before 17 months after birth are not useful for identification. The problem is that the bracelets or inks serve only for the period of permanence of the child in the hospital unit, and even during this period these IDs can be removed or altered. On the other hand, the DNA examination is proven to be efficient in the univocal identification of individuals, but it comes at high cost and cannot be used in real time applications, demanding sophisticated laboratory procedures.

The use of the iris as identification feature, even though it is increasingly used in adults [18], is a difficult method for infants, especially the premature, because they hardly open their eyes, they do not have the ability of looking into a scanning device, and touching their eyelids to collect an image could hurt them. Besides, the iris pattern only stabilizes after the child's second year [19]. The format of the ear is a biometric feature of easy acquisition, but possesses little discriminatory capacity and changes throughout the life of the individual $[18,20]$.

The work done by Rubisley P Lemes et al. demonstrate the use of palmprint using high resolution scanner on the database of 250 infant has the limitation of good quality image, high cost of recognition and highly intrusive [21]. Recently Tiwari et. al. tested the identification accuracy of infant using ear. The result on database of 210 infant using Geometrical Feature Extraction (GF) is $83.67 \%$, but the limitation of using ear biometrics for infant is pose and illumination covariates [22].

\section{ISSUES INVOLVED IN FACE RECOGNITION OF INFANTS}

The biggest challenge of Face recognition in infant is the availability of infant face data base and to the best of our knowledge there is not a single face data base of new born available as reported in the literature. To prepare a new face database of infant there are many issues involved in it. There are many challenges in the preparation of infant face database and the main challenge is the consent of parents and the cooperation of medical staff to prepare it. There is a need of participation of parents and the medical staff. It is really difficult to convince the parents for data acquisition in different poses; even some parents were due to privacy issues.

New born are highly non cooperative users and most of the time they are crying or sleeping. Thus it is really difficult to capture their face image because as soon as they are targeted for data acquisition they get disturbed and start crying as shown in sample infant image Fig.1. Soon after their birth some parents apply oil on the whole body of infant and this affects the texture quality of the infant image. During face image capturing a crucial problem is to decide an opportune time for face image acquisition. If an infant is uncomfortable due to medical illness or hunger then he/she will cry and ceaselessly move his/her feet, head or whole body. The task of their data acquisition becomes further challenging while they are sleeping due to closed eyes. It took about 40 to 45 minutes of time to prepare the favorable environment to capture the biometric data of a single infant.

Face recognition for adult is a long studied problem and several challenges have been identified by the researchers that are pose, expression, illumination, aging, and disguise. In case of infants, the challenges of aging and disguise are not manifested. However, pose and expression are two uncontrolled important covariates in case of infants. Since it is difficult to make the infants sit still and capture frontal face images with neutral expression, they can be considered as uncooperative users of face recognition. They may also exhibit different poses and expressions, especially if they become uncomfortable while photographing.

\section{INFANT DATABASE}

To prepare the face image database of infants 12 Megapixel Camera is used, and indoor images are captured at IMS-BHU, Varanasi Hospital-India. The data base acquisition of infants took more than five months to take sufficient number of subjects for the training and testing of different algorithms with slight variations of illumination, posing and expression conditions.

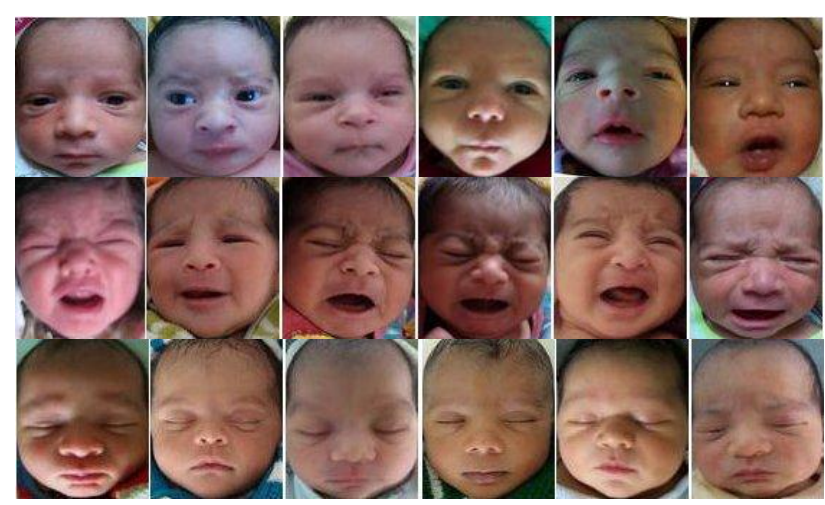

Fig. 1. Images of Infant from the Database

The infants face database is prepared in two different sessions. Data is collected within four hours of birth for the first session and second session data depends on the type of birth. If there is a case of normal birth then face image is collected after 20 hours otherwise in scissoring cases data is collected after 70 hours of the birth. In this data collection, the time for data acquisition is set according to the period that an infant stays in the hospital after birth. The sample image of face database is shown in Fig. 1. These images are captured without imposing any constraint on the targeted subjects or their surroundings.

\section{PROPOSED METHODOLOGY}

Principal component analysis (PCA) is a popular technique for dimensionality reduction. These techniques essentially select a subspace that retains most of that variation and consequently the similarity in the face space rarely determined. Much of the variation in the data is due to changes in illumination and expression. It has been found that PCA projection is optimal for reconstruction from a low dimensional basis; they may not be optimal from a discrimination point of view [23]. Contrary to this 
Fisher Linear Descriminant Analysis (FLDA) finds the projection of data in which the classes are most linearly separable. LDA is a method for high dimensional data analysis, as class labels are available in dataset.

The major limitation of PCA and ICA is that the distances between weights from faces of the same subject are greater than face weights from different people. To overcome this, a method called Fisherfaces [24], based on Linear Discriminant Analysis (LDA), attempts to find vectors that not only describe the data, but also best discriminate between classes of data. Given $c$ classes (infant) with the mean of class $j$ denoted by $\mu j$ and the $i^{\text {th }}$ infant face image in class $j$ denoted by $x_{i}^{j}$ a "within-class" scatter matrix $S_{w}$ and a "between-class" scatter matrix $S_{b}$ is calculated.

$$
\begin{gathered}
S_{b}=\sum_{j=1}^{c}\left(\mu_{j}-\mu\right)\left(\mu_{j}-\mu\right)^{T} \\
S_{w}=\sum_{j=1}^{c} \sum_{i=1}^{N j}\left(x_{i}^{j}-\mu_{j}\right)\left(x_{i}^{j}-\mu_{j}\right)^{T}
\end{gathered}
$$

To maximize the between class measure and simultaneously minimizing the within-class measure LDA produces a set of projection vector by using scatter matrices of infant image. Literature shows that LDA is often superior to PCA for well distributed classes in small datasets [24], Since LDA requires significantly more computation than PCA for large datasets. Eigen vector of the projection matrix is calculates as:

$$
U=\operatorname{eig}\left(S_{W}^{-1}\right)
$$

The linear transformation is given by a matrix $U$ whose columns are the eigenvectors of the above problem (i.e., called Fisher faces), has the projections with maximum class separability information are the eigenvectors corresponding to largest eigenvalues of. Because in practice $S_{W}$ is usually singular, the Fisher faces algorithm first reduces the dimensionality of the data with PCA and then applies FLD to further reduce the dimensionality to $C-1$. PCA smears the classes together, so it is no longer linearly separable. With FLD classification job is simplified as it achieves better between class scatter compare to PCA, though PCA achieves greater total scatter [23]. The $l^{2}$ norm $|X|$ is a vector norm defined for a complex vector

$$
X=\left[\begin{array}{c}
X_{1} \\
X_{2} \\
\vdots \\
X_{n}
\end{array}\right]
$$

9 Neurons by

$$
|X|=\sqrt{\sum_{k=1}^{n}\left|x_{k}\right|^{2}}
$$

where $x_{k}$ on the right denotes the complex modulus. The $l^{2}$ norm is the vector norm that is commonly found in vector algebra and vector operations, which it is commonly denoted $|X|$. Further, if desired, a more explicit notation $|X|_{2}$ is used to emphasize the distinction between the vector norm $|X|$ and complex modulus $|z|$ together with the fact the $l^{2}$ norm is just one of several possible types of norms. The absolute value sign indicating that a complex modulus is being taken on the right of the equation may be dropped. For example, the $l^{2}$ norm of the vector $X=\left(x_{1}, x_{2}, x_{3}\right)$ is given by

$$
|X|=\sqrt{x_{1}^{2}+x_{2}^{2}+x_{3}^{2}}
$$

The $l^{2}$ norm is also known as the Euclidean norm.

Test infant face is projected on face space and its features are compared with stored features either using $l^{2}$ norm, or test face features are given as an input to ANN or SVM classifiers, which finds out the most similar class for it.

\section{EXPERIMENTAL RESULTS}

We have used an intelligent classification technique such as back propagation neural network containing two hidden layers for classification. In our experiment, we have considered 9 neurons in input layer to receive the input from preprocessed infant image, shown in Fig. 2. in which we have taken 15 neurons in each hidden layers. We have trained the network for 5000 iterations with goal 0.00001 , and found enhancing result.

Motivation behind using SVM is that now-a-days, SVM are used as intelligent classifier that's why we have selected Support Vector Machine (SVM) classifier over Neural Network for classification. In SVM, a model is first created based on training samples. This model is then used to classify unknown data. Goal of SVM is to find out a hyper plane with largest class margin, to separate out given data elements. 


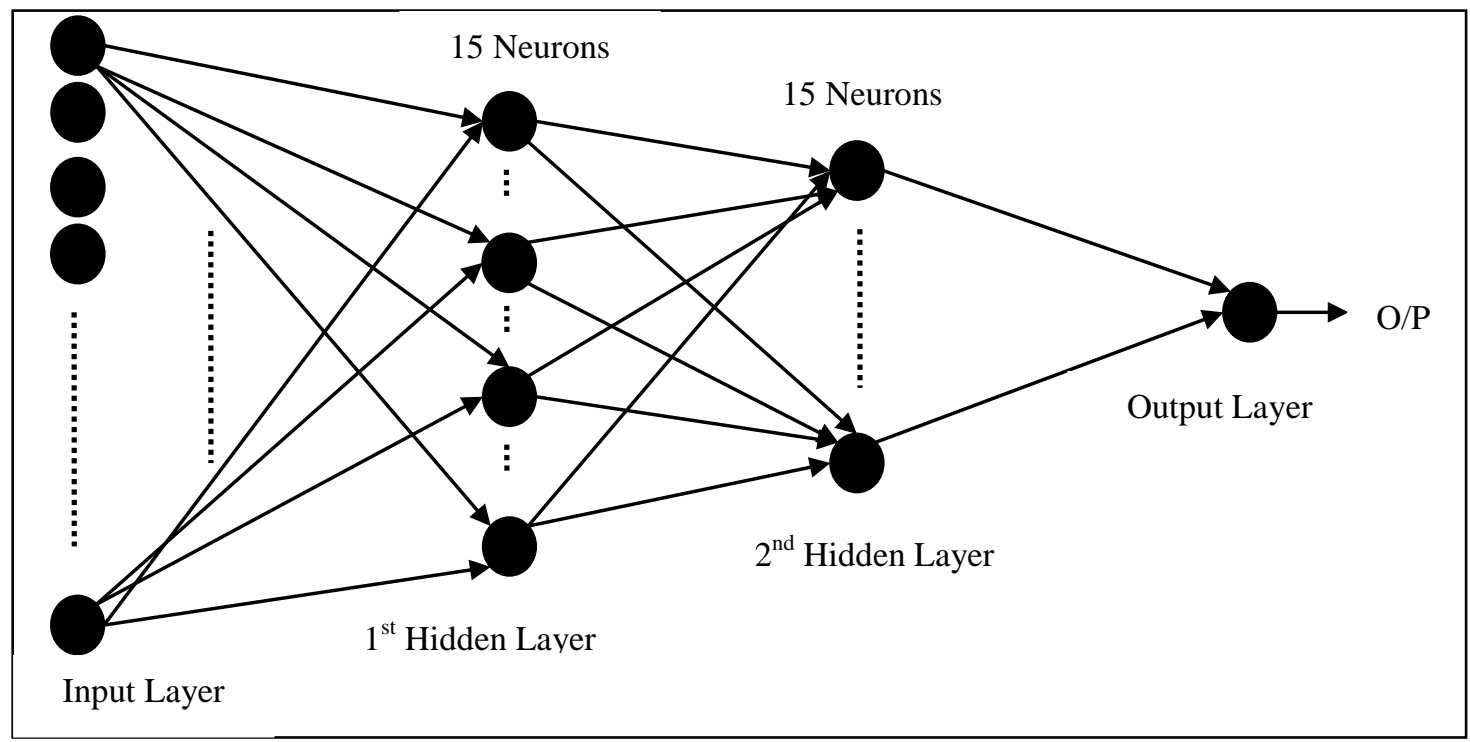

Fig. 2. Structure of Neural Network Used

Table 1: Results of FDA

\begin{tabular}{|l|l|l|l|l|}
\hline \multirow{2}{*}{$\begin{array}{l}\text { No. of Face } \\
\text { Per Subject }\end{array}$} & \multicolumn{4}{|c|}{ Accuracy (\%) } \\
\cline { 2 - 5 } & $\begin{array}{l}\text { Neural } \\
\text { Network }\end{array}$ & SVM & $l^{2}$-Norm & Average \\
\hline 1 & 63 & 86 & 91 & 80.00 \\
\hline 2 & 70 & 94 & 97 & 87.00 \\
\hline 3 & 72 & 95 & 98 & 88.33 \\
\hline 4 & 82 & 96 & 99 & 92.33 \\
\hline 5 & 90 & 98 & 99 & 95.67 \\
\hline 6 & 92 & 98 & 99 & 96.33 \\
\hline 7 & 93 & 99 & 99 & 97.00 \\
\hline 8 & 97 & 100 & 100 & 99.00 \\
\hline 9 & 97 & 99 & 100 & 98.67 \\
\hline 10 & 100 & 100 & 100 & 100.00 \\
\hline
\end{tabular}

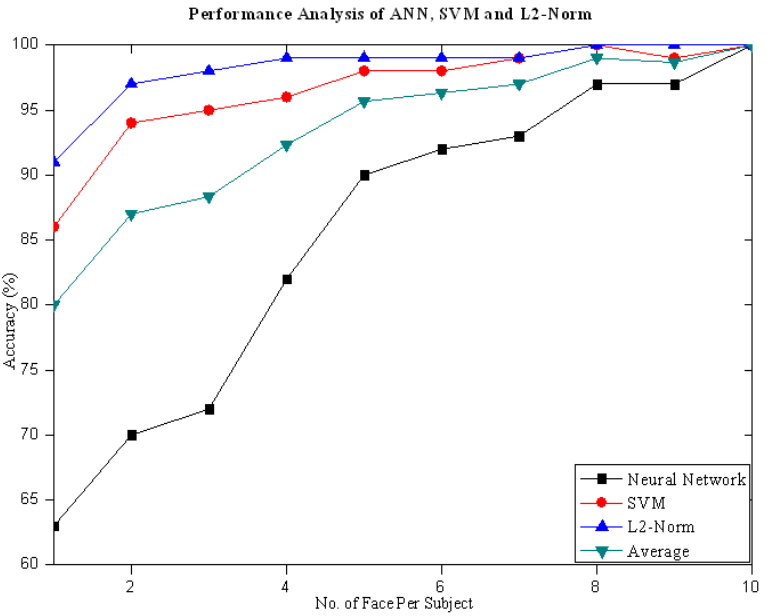

Fig. 3. Performance comparison between all three measures of FDA.

Table 1and Fig. 3 describes the results of the experiment carried out. As we have 10 classes (10 persons), with FDA we will have 9 features. These 9 features are used to classify the unknown data against three different classifiers, neural network, Support vector machine and $l^{2}$ norm. Compare to neural network and SVM classifiers, $l^{2}$ norm classifiers performance is high, neural network classifiers is better for small data base but as soon as data base size increase performance of neural network reduces. To obtain important feature we have merged all the three classifiers, neural network, Support vector machine and $l^{2}$ norm and find average which gives all important feature to recognize infant. Because infant is non-cooperative user and most of the time they are sleeping so extracting feature from infant image is a challenging task. Experimental statics also concluded that $l^{2}$ norm performance is superior to other classifier.

\section{DISCUSSION}

Face recognition of infant has been an unexplored area in the face recognition domain and it poses ethical, social and engineering challenges. Swapping and abduction of infant probably garner such a strong negative response because they are things that parents fear the most which seem difficult or impossible to check or stop. Baby switching and abduction are very serious problems across the world which need immediate attention. Several biometric and non-biometric techniques have been evaluated to reduce the number of such incidences. This research performs a study on using automatic face recognition for identifying infants using intelligent techniques. In future more advanced algorithms can be implemented to improve the recognition accuracy. With proper training, hospital personal can assist law enforcement agency efforts by successfully and consistently obtaining face image of infant. Face recognition can be friendly and cost effective solution for identification of infants for short period of time if the performance of automatic matching algorithm is satisfactory.

\section{CONCLUSION}

The domain of biometrics is a new and exciting area of information science research directed toward understanding of traits and methods for accurate and reliable personal information representation for subsequent decision making and matching. Recent years have seen a significant increase in research activity directed at understanding all aspects of biometric information system representation and utilization for decision-making 
support, for use by public and security services, and for understanding the complex processes behind biometric matching and recognition. This paper is specifically focused on understanding the complex mechanisms employed to identify infant using novel intelligent technique. Neural network separates classes through only single lines, while SVM separates classes through fuzzier boundary Moreover, with 40 classes; neural network is not able to find generalized mapping function, which can classify all the data correctly. From results, we can conclude that SVM out weights the performance of the Neural network with improvement of more than $10 \%$.

\section{ACKNOWLEDGEMENT}

We would like to thank Prof. B. M. Singh and Dr. Niraj Srivastava (Department of Kaumar Bharitya, Faculty of Aurveda, Institute of Medical Science, BHU, Varanasi) for their help and cooperation in preparing the face database of infants.

\section{REFERENCES}

[1] "http: //www.amf or.net/stolenbabies.html", Last accessed on May 25, 2011.

[2] "http://www.missingkids.com/enus/documents/infantabdu ctionstats.pdf', Last accessed on June 4, 2011.

[3] J.E. Gray, G. Suresh, R. Ursprung, W.H. Edwards, J. Nickerson, and P.H. Shinno, "Patient Misidentification in the neonatal intensive care unit: Quantification of risk", Pediatrics, vol. 117, pp. e46-e47, 2006

[4] A. Ross, K. Nandakumar, and A.K. Jain, "Handbook of Multibiometrics", Springer, New York, 2006

[5] Anastasi, J. S., and Rhodes, M. G., "An own-age bias in face recognition for children and older adults". Psychonomic Bulletin \& Review, 12, 1043-1047.2005.

[6] Anastasi, J. S., \& Rhodes, M. G., "Evidence for an ownage bias in face recognition". North American Journal of Psychology, 8, 237-253, 2006.

[7] Lamont, A. C., Stewart-Williams, S., \& Podd, J., "Face recognition and aging: Effects of target age and memory load". Memory \& Cognition, 33, 1017-1024, 2005.

[8] Rhodes, G., Hayward, W. G., \& Winkler, C., "Expert face coding: Configural and component coding of own-race and other-race faces". Psychonomic Bulletin \& Review, 13, 499-505, 2006.

[9] Thompson, J. E., Clark, D. A., Salisbury, B., and Cahill, J., "Footprinting the newborn: not cost-effective". Journal of Pediatrics, 99:797-798, 1981.

[10] M.E. Stapleton, "Best foot forward: Infant footprints for personal identification", Law Enforcement Bulletin 63, FBI, 1999.

[11] Galton, F., "Finger prints of young children". British Association for the Advancement of Science, 1899.
[12] K.S. Shepard, T. Erickson, and H. Fromm, "Limitations of footprinting as a means of infant identification", Pediatrics, vol. 37, no. 1, 1966.

[13] N.T.R. Pela, M.V. Mamede, and M.S.G. Tavares, "Analise crtica de impressoes plantares de recemnascidos", Revista Brasileira de Enfermagem, vol. 29, pp. 100-105, 1975.

[14] D. Weingaertner, O.R.P. Bellon, M.N.L. Cat, and L. Silva, "Newborn's biometric identification: Can it be done?" in International Joint Conference on Computer Vision, Imaging and Computer Graphics Theory and Applications, 2008.

[15] C. Fields, C.F. Hugh, C.P. Warren, and M. Zimberoff, "The ear of the newborn as an identification constant", Journal of Obstetrics and Gynecology, vol. 16, pp. 98101,1960

[16] S.Z. Li and A.K. Jain, "Handbook of Face Recognition", Springer, New York, 2004.

[17] J. Daugman, "New methods in iris recognition", IEEE Transactions on Systems, Man and Cybernetics B, vol. 37, no. 5, pp. 1167-1175, 2007.

[18] Bolle, R., Connell, J., Pankanti, S., Ratha, N., and Senior, A., "Guide to Biometrics". Springer Verlag, 2003.

[19] Jain, A. K., Ross, A., and Prabhakar, S. "An introduction to biometric recognition". IEEE Trans. Circuits and Systems for Video Technology, 14(1):4-20, 2004.

[20] Victor, B., Bowyer, K., and Sarkar, S., "An evaluation of face and ear biometrics". Proceedings. 16th International Conference on Pattern Recognition, 01:10429, 2002.

[21] Rubisley P Lemes, Olga R P Bellon, Luciano Silva, Anil $\mathrm{K}$ Jain, "Biometric Recognition of Newborns: Identification using Palmprints", International Joint Conference on Biometrics, October 11-13, Washington DC USA, 2011

[22] Shrikant Tiwari, Aruni Singh and Sanjay K Singh, "Newborn's ear recognition: Can it be done?", International Conference on Image Information Processing (ICIIP), 3-5 Nov. JUIT India, 2011.

[23] B J Oh, "Face Recognition using Radial Basis Function Networkbased on LDA" World Academy of Science and Technology 2005.

[24] Peter N. Belhumeur, Jo ao P. Hespanha, and David J. Kriegman, "Eigenfaces vs. Fisherfaces: RecognitionUsing Class Specific Linear Projection", IEEE Transaction on Pattern Analysis and Machine Intelligence, Vol. 19, No. 7, pp. 711-720, July - 1997.

[25] R. Duda and P. Hart, Pattern Classification and Scene Analysis. New York: Wiley, 1973. 